\title{
CONSTRUÇÃO DE EMPACOTAMENTOS ESFÉRICOS VIA CÓDIGOS DE CLASSES LATERAIS GENERALIZADOS
}

\author{
Antonio de Andrade e Silva ${ }^{1}$ e Reginaldo Palazzo Jr ${ }^{2,3}$ \\ ${ }^{1}$ Departamento de Matemática, CCEN - UFPb, 58.059-900, J. Pessoa, Pb. \\ ${ }^{2}$ Departamento de Telemática, FEEC - UNICAMP, CP 6101, 13081-970, Campinas, SP, Brasil. \\ palazzo@dt.fee.unicamp.br.
}

\begin{abstract}
Resumo - Neste trabalho propomos uma construção de empacotamentos esféricos tendo como base códigos de bloco sobre o grupo $\mathbb{Z}_{q}$. Esta construção é uma generalização da construção proposta por Costa e Silva e Palazzo [4]. Tabela e exemplos são incluídos com os melhores empacotamentos esféricos obtidos através desta construção. Nas dimensões 68 e 72, um novo recorde de densidades foi alcançado.
\end{abstract}

Abstract - We propose a construction scheme for sphere packing arrangements based on block codes over the $\mathbb{Z}_{q}$ group. This scheme is a generalization of the one proposed by Costa e Silva and Palazzo [4]. A table and examples are included with results from the best sphere packings obtained with this construction. In dimensions 68 and 72 a new density record is achieved.

Palavras-chave: Empacotamento de esferas, códigos de bloco, códigos sobre grupos, códigos de classes laterais.

\section{INTRODUÇÃO}

A primeira proposta de construção de um reticulado $\Lambda$ em $\mathbb{R}^{N}$ utilizando um código linear foi apresentada por Leech e Sloane [10]. Desde então, novos métodos de construção de reticulados utilizando códigos lineares foram apresentados, [1], [4], [7], [8], [9], e [12]. Para maiores detalhes sobre empacotamentos esféricos e reticulados sugerimos ao leitor a referência [2].

Os métodos propostos invariavelmente utilizam do seguinte procedimento. Seja $C=[N, k, d]_{p}$ um código linear sobre $\mathbb{Z}_{p} \cong G F(p)$, onde $p$ é um número primo. Se identificarmos $\mathbb{Z}_{p}$ com o subconjunto $\mathbb{N}_{p}=\{0,1, \ldots, p-1\} \subset \mathbb{Z}$, então

$$
\Lambda=\bigcup_{\mathbf{c} \in C}\left(l(\mathbf{c})+p \mathbb{Z}^{N}\right)
$$

é um reticulado em $\mathbb{R}^{N}$ consistindo da união de $p^{k}$ classes laterais à esquerda de $p \mathbb{Z}^{N}$, onde $\mathbb{Z}_{p} \simeq \mathbb{Z} / p \mathbb{Z}$ é um grupo de rótulos, $l: \mathbb{Z}_{p}^{N} \longrightarrow[\mathbb{Z} / p \mathbb{Z}]^{N}$ e c $\in C$.

Os principais métodos de construção de novos empacotamentos esféricos, usando reticulados conhecidos, consistem da união de um número finito de elementos de uma partição. Mais precisamente, sejam $\Gamma$ e $\Lambda$ reticulados em $\mathbb{R}^{N}$ tal que $\Lambda$ é um subreticulado de $\Gamma$. Então $\Lambda$ induz uma partição $\Gamma / \Lambda$ de $\Gamma$ em $[\Gamma: \Lambda]$ classes laterais de $\Lambda$. Seja $[\Gamma / \Lambda]$ um sistema de representantes de classes laterais de $\Lambda$ em $\Gamma$. Dado $H \subset[\Gamma / \Lambda], \mathbb{L}_{N}=H+\Lambda$ é um empacotamento esférico em $\mathbb{R}^{N}$ e é um reticulado se, e somente se, $H$ é um subgrupo de $[\Gamma / \Lambda]$ com a operação de adição módulo $\Lambda$. De um modo geral, $\mathbf{L}_{N n}=H+\Lambda^{n}$ é um empacotamento esférico em $\mathbb{R}^{N n}$ e é um reticulado se, e somente se, $H$ é um subgrupo de $\left[\Gamma^{n} / \Lambda^{n}\right]$ com a operação de adição módulo $\Lambda^{n}$, onde $\Gamma^{n} / \Lambda^{n}$ é isomorfo a $(\Gamma / \Lambda)^{n}$.

Por exemplo, sejam $\Gamma=\mathbb{Z}$ e $\Lambda=2 \mathbb{Z}$ reticulados em $\mathbb{R}$ tal que $\Lambda$ é um subreticulado de $\Gamma$ e $[\Gamma / \Lambda]=\{0,1\}$ um sistema de representantes de classes laterais de $\Lambda \mathrm{em}$ $\Gamma$. Então $\mathbf{L}_{4}=H+\Lambda^{4}$ é um reticulado em $\mathbb{R}^{4}$ que tem $\Lambda^{4}$ como um subreticulado e por sua vez é um subreticulado de $\Gamma^{4}$, onde o subgrupo selecionado é dado por $H=$ $\{(0,0,0,0),(1,1,0,0)\} \subset[\Gamma / \Lambda]^{4}$. Sob esta construção, $\mathbf{L}_{4}$ apresenta um ganho de codificação fundamental de $1.51[d B]$,

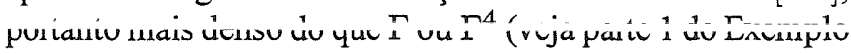
1).

Chamamos a atenção para o fato de que a construção de empacotamentos esféricos da forma $\mathbf{L}_{N n}$ que sejam mais densos do que $\Gamma$ para a utilização em esquemas de quantização e/ou codificação de canais com ruído gaussiano branco aditivo com faixa limitada, torna-se impraticável quando a ordem da partição $\Gamma / \Lambda$ for muito grande. Infelizmente, não se conhece nenhum método sistemático de selecionar o subgrupo $H$ de $[\Gamma / \Lambda]^{n}$ de modo que o empacotamento esférico $\mathbf{L}_{N n}=H+\Lambda^{n}$ seja mais denso do que $\Gamma^{n}$.

Seja $r$ a ordem da partição $\Gamma / \Lambda$ e $r=\prod_{i=0}^{m-1} q_{i}$, onde $q_{i}=p_{i}^{n_{i}}, \operatorname{com} p_{i}$ números primos distintos, $0 \leq i \leq m-1 \mathrm{e}$ $n_{0}+\cdots+n_{m-1} \leq N$. Como $\Gamma / \Lambda$ é um grupo comutativo finito, existe um refinamento da partição $\Gamma / \Lambda$ em uma cadeia de partições de $m$-níveis e $q_{i}$-maneiras, [5],

$$
\Lambda_{0} / \Lambda_{1} / \cdots / \Lambda_{m-1} / \Lambda_{m}
$$

onde

$$
\Lambda_{0}=\Gamma, \Lambda_{m}=\Lambda, \Lambda_{i}=\left[\Lambda_{i} / \Lambda_{i+1}\right]+\Lambda_{i+1}
$$

e

$$
\left|\Lambda_{i} / \Lambda_{i+1}\right|=q_{i}, 0 \leq i \leq m-1 .
$$

\footnotetext{
${ }^{3}$ Este trabalho foi financiado em parte pela Fundação de Amparo à Pesquisa do Estado de São Paulo, FAPESP, No. 95/4720-8, e em parte pelo Conselho Nacional de Desenvolvimento Científico e Tecnológico, CNPq, No. 301416/85-0. 
Assim, um método alternativo de construção de empacotamentos esféricos da forma $\mathbf{L}_{N n}=H+\Lambda_{m}^{n}$ é considerar o subconjunto $H$ de $\left[\Lambda_{0} / \Lambda_{m}\right]^{n}$ definido por $H=\sum_{i=0}^{m-1} H_{i}$, onde $H_{i}$ são subconjuntos de $\left[\Lambda_{i} / \Lambda_{i+1}\right]^{n}, 0 \leq i \leq m-1$. E claro que $\mathbb{L}_{N n}$ é um reticulado se, e somente se, cada $H_{i}$ é um subgrupo de $\left[\Lambda_{i} / \Lambda_{i+1}\right]^{n}, 0 \leq i \leq m-1$. Uma vez que $\Lambda_{i} / \Lambda_{i+1}$ é isomorfo ao grupo de rótulos $\mathbb{Z}_{q_{i}}, 0 \leq i \leq m-1$, os conjuntos $H_{i}$ podem ser identificados com códigos $C_{i}$ sobre $\mathbb{Z}_{q_{i}}$. Assim, cada palavra-código $\mathbf{c}_{i} \in C_{i}$ será identificada com um elemento $h_{i} \in H_{i}$ e $\sum_{i=0}^{m-1} h_{i}$ é o representante de alguma classe lateral de $\Lambda_{m}^{n}$ em $\Lambda_{0}^{n}$. Portanto, $L_{N n}$ é a união de todas as classes laterais de $\Lambda_{m}^{n}$.

Este trabalho é constituído da seguinte forma. Na Seção 2, apresentamos uma construção multicamada, que estende a proposta de [4], com o objetivo de obter empacotamentos esféricos com o maior ganho de codificação fundamental possível em $\mathbb{R}^{N n}$. Na Seção 3, com o propósito de completeza, um algoritmo de decodificação com distância limitada é exibido para a decodificação dos reticulados obtidos através desta construção. Na Seção 4, como resultado da construção sendo proposta, apresentamos empacotamentos esféricos conhecidos e novos, onde nas dimensões 68 e 72 um novo recorde de densidades foi alcançado.

\section{CONSTRUÇÃO DE EMPACOTAMEN- TOS ESFÉRICOS}

Os resultados desta seção estendem a proposta de [4], para qualquer número $r$, onde $r=\prod_{i=0}^{m-1} q_{i}$ é a decomposição de $r$ em fatores primos distintos. Com o propósito de completeza, apresentamos as provas de todos os resultados que foram estendidos. Para maiores detalhes, [3].

Sejam $\Gamma$ e $\Lambda$ reticulados em $\mathbb{R}^{N}$ tal que $\Lambda$ é um subreticulado de $\Gamma$. Então $\Lambda$ induz uma partição $\Gamma / \Lambda$ de ordem $r$ e $r \Gamma \subseteq \Lambda$. Se

$$
[\Gamma / \Lambda]=\left\{\sum_{i=0}^{m-1} a_{i} \mathbf{g}_{i}: a_{i} \in \mathbb{N}_{q_{i}}\right\},
$$

onde $\mathbf{g}_{i} \in \Gamma, \mathbf{g}_{i} \notin \Lambda$ e $\mathbb{N}_{q_{i}}=\left\{0, \ldots, q_{i}-1\right\}$, é um sistema de representantes de classes laterais fixo de $\Lambda \mathrm{em} \Gamma$. Então uma cadeia de partições

$$
\Lambda_{0} / \Lambda_{1} / \cdots / \Lambda_{m-1} / \Lambda_{m},
$$

com $m$-níveis e $q_{i}$-maneiras, pode ser definida como um refinamento de $\Gamma / \Lambda$, fazendo

$$
\Lambda_{0}=\Gamma, \Lambda_{m}=\Lambda \text { e } \Lambda_{i}=\left[\Lambda_{i} / \Lambda_{i+1}\right]+\Lambda_{i+1},
$$

onde

$$
\left[\Lambda_{i} / \Lambda_{i+1}\right]=\left\{a_{i} \mathrm{~g}_{i}: a_{i} \in \mathbb{N}_{q_{i}}\right\}, \mathrm{g}_{i} \in \Lambda_{i}, \mathrm{~g}_{i} \notin \Lambda_{i+1}
$$

e

$$
\left|\Lambda_{i} / \Lambda_{i+1}\right|=q_{i}, 0 \leq i \leq m-1,
$$

$\operatorname{com} \mathbb{Z}_{q_{i}} \simeq \Lambda_{i} / \Lambda_{i+1}, 0 \leq i \leq m-1$ um grupo de rótulos.
Definição 1 Sejam $\Lambda_{0} / \Lambda_{1} / \cdots / \Lambda_{m-1} / \Lambda_{m}$ uma cadeia de partições de reticulados em $\mathbb{R}^{N}$, com m-níveis e $q_{i}$-maneiras, e $C_{0}, \ldots, C_{m-1}$ códigos de bloco sobre $\mathbb{Z}_{q_{i}}$ cada um de comprimento n. Fixado $\alpha_{i} \in\left[\Lambda_{i} / \Lambda_{i+1}\right]-\{0\}, 0 \leq i \leq$ $m-1$, define-se um empacotamento esférico $\mathbb{L}_{N n}$ em $\mathbb{R}^{N n}$ como o conjunto

$$
\begin{gathered}
\mathbf{L}_{N n} \triangleq\left\{\sum_{i=0}^{m-1}\left(\mathbf{c}_{i} \otimes \alpha_{i}\right): \mathbf{c}_{i} \in C_{i}, 0 \leq i \leq m-1\right\}+\Lambda_{m}^{n} \\
=\bigcup_{\mathbf{c}_{i} \in C_{i}}\left(\sum_{i=0}^{m-1}\left(\mathbf{c}_{i} \otimes \alpha_{i}\right)+\Lambda_{m}^{n}\right)
\end{gathered}
$$

onde para cada $\mathbf{c}_{i}=\left(c_{i 1}, \ldots, c_{i n}\right) \in C_{i}, \mathbf{c}_{i} \otimes \alpha_{i} \triangleq$ $\left(c_{i 1} \alpha_{i}, \ldots, c_{i n} \alpha_{i}\right)$, com as palavras-código $\mathbf{c}_{i}$ vistas como um vetor real de componentes $c_{i j} \in \mathbb{N}_{q_{i}}: 0 \leq i \leq$ $m-1,: 1 \leq j \leq n$ e a união sendo sobre todos os códigos $C_{0}, \ldots, C_{m-1}$.

Chamamos a atenção de que o empacotamento esférico $\mathbb{L}_{N n}$ é periódico, no sentido de que ele é invariante sob deslocamento de múltiplos inteiros dos vetores básicos de $\Lambda_{m}^{n}$. O arranjo periódico $\mathbb{L}_{N n}$ é chamado de código de classe lateral generalizado [6], e denotado por

$$
\mathbf{L}_{N n}=\sum_{i=0}^{m-1}\left(C_{i} \otimes\left[\Lambda_{i} / \Lambda_{i+1}\right]\right)+\Lambda_{m}^{n}
$$

Dados $\mathbf{l} \in \mathbb{L}_{N n}, \mathbf{l}=\sum_{i=0}^{m-1}\left(\mathbf{c}_{i} \otimes \alpha_{i}\right)+\lambda$, com

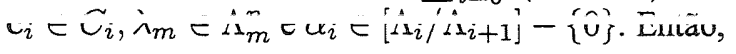

$$
\begin{aligned}
\mathbb{I} & =\sum_{i=0}^{m-1}\left(c_{i 1} \alpha_{i}, \ldots, c_{i n} \alpha_{i}\right)+\left(\lambda_{m 1}, \ldots, \lambda_{m n}\right) \\
& =\left(\sum_{i=0}^{m-1}\left(c_{i 1} \alpha_{i}\right), \ldots, \sum_{i=0}^{m-1}\left(c_{i n} \alpha_{i}\right)\right)+\left(\mathbf{v}_{1} D, \ldots, \mathbf{v}_{n} D\right)
\end{aligned}
$$

Portanto, $1=\left(\mathbf{u}_{1} B, \ldots, \mathbf{u}_{n} B\right)+V D$, onde $\mathbf{u}_{k}=$ $\left(c_{0 k}, \ldots, c_{(m-1) k}\right), 1 \leq k \leq n$, é a $m$-upla formada da $k$ ésima componente de cada palavra-código $\mathrm{c}_{i} \in C_{i}, 0 \leq i \leq$ $m-1$, isto é, $\mathbf{u}_{k}$ é um rótulo de alguma classe lateral à esquerda de $\Lambda_{m}$ em $\Lambda_{0}, B$ é uma matriz $m \times N$ cujas linhas são os vetores $\alpha_{0}, \ldots, \alpha_{m-1}$, isto é, $B=\left\{\mathbf{g}_{i}: 0 \leq i \leq m-1\right\}$, pois $\alpha_{i}=a \mathbf{g}_{i}, a \in \mathbb{N}_{q_{i}}-\{0\}, V=\left\{\mathbf{v}_{k}: 1 \leq k \leq n\right\}$ é uma matriz $m \times N$ cujas linhas são os vetores $\mathbf{v}_{k} \in \mathbb{Z}^{N}, 1 \leq$ $k \leq n$, e $D=M_{m}^{t}$, onde $M_{m}$ é uma matriz geradora para o reticulado $\Lambda_{m}$. Assim,

$$
1=U B+V D,
$$

onde $U=\left\{\mathfrak{u}_{k}: 1 \leq k \leq n\right\}$ é uma matriz $n \times m$ cujas linhas são os vetores $\mathfrak{u}_{k} \in \mathbb{N}_{q_{i}}^{m}$. Isto significa que a $i$-ésima linha do produto $U B$ é uma combinação linear das linhas de $B$ com escalares da $i$-ésima linha de $U$. Por essa razão, vamos considerar

$\mathbb{L}_{N n}=\left\{\sum_{i=0}^{m-1}\left(\mathbf{c}_{i} \otimes \mathbf{g}_{i}\right): \mathbf{c}_{i} \in C_{i}, 0 \leq i \leq m-1\right\}+\Lambda_{m}^{n}$. 
Isto exibe explicitamente $\mathbb{L}_{N n}$ como a união de $\prod_{i=0}^{m-1}\left|C_{i}\right|$ classes laterais de $\Lambda_{m}^{n}$, onde $\left|C_{i}\right|$ é a cardinalidade do código $C_{i}$. Em particular, se $q_{i}=q$ e os códigos $C_{i}, 0 \leq i \leq m-1$, são lineares, isto é, subgrupos de $\mathbb{Z}_{q}^{n}$, então o empacotamento esférico $\mathbb{L}_{N n}$ é um reticulado em $\mathbb{R}^{N n}$ (Teorema 2). Neste caso,

$$
\mathbf{c}_{i}+\mathbf{c}_{j}=\mathbf{c}_{i} \oplus \mathbf{c}_{j}+q\left(\mathbf{c}_{i} \circ \mathbf{c}_{j}\right),
$$

onde "+" é a soma ordinária em $\mathbb{R}^{N}$, " $\oplus$ " é soma em $\mathbb{Z}_{q}$ e "o" é definida por

$$
\mathbf{c}_{i} \circ \mathbf{c}_{j} \triangleq\left(\left\lfloor\frac{1}{q}\left(c_{i 1}+c_{j 1}\right)\right\rfloor, \ldots,\left\lfloor\frac{1}{q}\left(c_{i n}+c_{j n}\right)\right\rfloor\right),
$$

sendo $\lfloor x\rfloor$ o maior inteiro menor ou igual a $x$. No caso em que $q=2$, temos

$$
\left\lfloor\frac{1}{2}\left(c_{i k}+c_{j k}\right)\right\rfloor=c_{i k} c_{j k}, 1 \leq k \leq n .
$$

Note que, quando $\mathbb{Z}_{q}$ é substituído pelo corpo de Galois $\mathbb{F}_{q}$, a soma acima apresenta restrições, pois neste caso a soma depende da característica do corpo. Uma alternativa é considerar os códigos $C_{i}$ como sendo quasilineares, [11]. Se $q_{i}=q$ e os códigos $C_{i}, 0 \leq i \leq m-1$, são lineares, então temos o seguinte resultado.

Teorema 2 Se $q_{i}=q$ e os códigos $C_{i},: 0 \leq i \leq m-1$, são lineares, então $\mathbf{L}_{N n}$ é um reticulado em $\mathbb{R}^{N n}$.

Prova. É claro que $0 \in \mathbb{L}_{N n}$. Dado $\mathbf{l}=U B+\lambda_{m} \in \mathbf{L}_{N n}$.

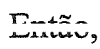

$$
\mathbf{l}=((1-q) U) B+(q U) B+\lambda_{m} .
$$

Assim, existe $\mathbf{l}^{\prime}=((q-1) U) B-\lambda_{m}^{\prime} \in \mathbb{L}_{N n}$, onde $\lambda_{m}^{\prime}=(q U) B+\lambda_{m} \in \Lambda_{m}^{n}$, pois $q \Lambda_{0} \subseteq \Lambda_{m}$, tal que $\mathbb{l}+1^{\prime}=0$. Dados $1,1^{\prime} \in \mathbb{L}_{N n},: 1=U B+\lambda_{m}$ e $1^{\prime}=U^{\prime} B+\lambda_{m}^{\prime}$. Então,

$$
\begin{aligned}
1+\mathbf{l}^{\prime} & =U B+\lambda_{m}+U^{\prime} B+\lambda_{m}^{\prime} \\
& =\left(U+U^{\prime}\right) B+\lambda_{m}+\lambda_{m}^{\prime}
\end{aligned}
$$

Como $U+U^{\prime}=U \oplus U^{\prime}+q\left(U \circ U^{\prime}\right)$ e $\mathbf{c}_{i} \oplus \mathbf{c}_{i}^{\prime}=\overline{\mathbf{c}}_{i} \in C_{i}$ temos que

$$
\mathbf{I}+\mathbf{l}^{\prime}=\bar{U} B+\bar{\lambda}_{m} \in \mathbb{L}_{N n},
$$

pois, $\bar{U}=U \oplus U^{\prime}$ e $\bar{\lambda}_{m}=q\left(U \circ U^{\prime}\right) B+\lambda_{m}+\lambda_{m}^{\prime} \in \Lambda_{m}^{n}$. Portanto, $\mathbb{L}_{N n}$ é um subgrupo de $\mathbb{R}^{N n}$.

O teorema a seguir determina uma cota da mínima distância euclidiana quadrática dos empacotamentos esféricos da Definição 1.

Teorema $3 \min _{0 \leq i \leq m-1}\left\{d_{H}\left(C_{i}\right) d_{\min }^{2}\left(\Lambda_{i}\right), d_{\min }^{2}\left(\Lambda_{m}\right)\right\} \leq$ $d_{\min }^{2}\left(\mathrm{~L}_{N n}\right) \leq d_{\min }^{2}\left(\Lambda_{m}\right)$. Em particular, se $C_{0} \subseteq \cdots \subseteq$ $C_{m-1} e q_{i}=q, 0 \leq i \leq m-1$, então

$$
d_{\min }^{2}\left(L_{N n}\right)=\min _{0 \leq i \leq m-1}\left\{d_{H}\left(C_{i}\right) N\left(\Lambda_{i}\right), d_{\min }^{2}\left(\Lambda_{m}\right)\right\} .
$$

Corolário 4 Se $C_{0} \subseteq \cdots \subseteq C_{m-1}$ e $q_{i}=q, 0 \leq$ $i \leq m-1$, então dados $1 \in \mathbb{L}_{N n},: 1=U B+\lambda_{m}$ e $\mathbf{u}_{k} \neq 0$, para algum $k,: 1 \leq k \leq n$, temos que $\mathrm{N}(\mathrm{l})=d_{\min }^{2}\left(\mathbf{L}_{N n}\right)$ se, e somente se, $w_{H}\left(\mathbf{c}_{i}\right)=d_{H}\left(C_{i}\right)$ $e d_{\min }^{2}\left(\mathbf{L}_{N n}\right)=d_{H}\left(C_{i}\right) d_{\min }^{2}\left(\Lambda_{i}\right), 0 \leq i \leq m-1$, onde $\mathbf{u}_{k}=\left(c_{0 k}, \ldots, c_{m-1 k}\right) \neq 0$ implica que $\mathbf{c}_{i} \neq 0$ para algum $i,: 0 \leq i \leq m-1$.

O cálculo do coeficiente de erro $E\left(\mathbb{L}_{N n}\right)$ do reticulado $\mathbb{L}_{N n}$ em $\mathbb{R}^{N n}$ é feito através da função Téta de Jacobi, $\theta_{\mathrm{L}_{N n}}(z),[2]$, isto é,

$$
\theta_{\mathbf{L}_{N n}}(z)=\sum_{j=1}^{K} \sum_{\lambda \in \Lambda_{m}^{n}} q^{\mathrm{N}\left(\lambda+\mathbf{h}_{j}-\mathbf{h}_{1}\right)},
$$

onde $q=e^{i \pi z}, \mathbf{h}_{j} \in\left\{\sum_{i=0}^{m-1}\left(\mathbf{c}_{i} \otimes \mathbf{g}_{i}\right): \mathbf{c}_{i} \in C_{i}\right\}$ e $K=$ $\prod_{i=0}^{m-1}\left|C_{i}\right|$, pois $\mathbf{L}_{N n}$ é um empacotamento periódico.

Com o propósito de comparação, será conveniente normalizar o coeficiente de erro para duas dimensões, isto é,

$$
\tilde{E}\left(\mathbb{L}_{N n}\right) \triangleq \frac{2}{N n} E\left(\mathbf{L}_{N n}\right) .
$$

Como o empacotamento esférico $\mathbb{L}_{N n}$ em $\mathbb{R}^{N n}$ é periódico, a densidade $\mathbf{L}_{N n}$ é dada por

$$
\Delta\left(\mathbb{L}_{N n}\right) \triangleq \prod_{i=0}^{m-1}\left|C_{i}\right| \frac{V\left(\mathbb{E}_{\rho}(0)\right)}{V\left(\Lambda_{m}\right)^{n}},
$$

onde $\rho=\frac{1}{2} d_{\min }\left(\mathbf{L}_{N n}\right)$. Consequentemente, a densidade de rentro do Tr.... ó dada nor

$$
\delta\left(\mathbb{L}_{N n}\right) \triangleq \prod_{i=0}^{m-1}\left|C_{i}\right| \frac{\rho^{N n}}{V\left(\Lambda_{m}\right)^{n}}
$$

O ganho de codificação fundamental do empacotamento esférico $\mathbb{L}_{N n}$ em $\mathbb{R}^{N n}$ é dado, [6], por

$$
\gamma\left(\mathbf{L}_{N n}\right) \triangleq \frac{d_{\min }^{2}\left(\mathbb{L}_{N n}\right)}{\left[V\left(\mathbf{L}_{N n}\right)\right]^{\frac{2}{N n}}},
$$

onde $V\left(\mathbb{L}_{N n}\right)$ é o volume da região de Voronoi em $\mathbb{L}_{N n}$. Para calcular o ganho de codificação fundamental devemos encontrar o volume de uma região de Voronoi de $\mathbb{L}_{N n}$. Como $\mathbf{L}_{N n}$ é periódico, basta considerar apenas um período, o qual corresponde a uma região congruente à região fundamental básica de $\Lambda_{m}^{N n}$, pois $\mathbb{L}_{N n}$ é a união de $\prod_{i=0}^{m-1}\left|C_{i}\right|$ classes laterais à esquerda de $\Lambda_{m}^{N n}$. Assim, esta região contém $\prod_{i=0}^{m-1}\left|C_{i}\right|$ centros de esferas, uma vez que as regiões de Voronoi destes centros cobrem esta região. Desse modo, temos que

$$
V\left(\mathbb{L}_{N n}\right)=\frac{V\left(\Lambda_{m}^{n}\right)}{\prod_{i=0}^{m-1}\left|C_{i}\right|}=\frac{V\left(\Lambda_{m}\right)^{n}}{\prod_{i=0}^{m-1}\left|C_{i}\right|}
$$

Portanto, o ganho de codificação fundamental de $\mathbb{L}_{N n}$ é dado por

$$
\gamma\left(\mathbb{L}_{N n}\right)=\left(\prod_{i=0}^{m-1}\left|C_{i}\right|\right)^{\frac{2}{N n}} \frac{d_{\min }^{2}\left(\mathbb{L}_{N n}\right)}{V\left(\Lambda_{m}\right)^{\frac{2}{N}}}
$$


Neste caso, temos a seguinte relação

$$
\delta\left(\mathbf{L}_{N n}\right)=\left(\frac{\gamma\left(\mathbf{L}_{N n}\right.}{4}\right)^{\frac{N n}{2}}
$$

Note que, o ganho de codificação fundamental $\gamma\left(\mathbb{L}_{N n}\right)$ mede uma componente do ganho de potência que pode ser alcançado usando uma constelação de sinais $S$ com base no reticulado $\mathrm{L}_{N n}$ relativo a uma constelação de sinais com base em $\mathbb{Z}^{N n}$.

\section{DECODIFICAÇÃO}

Com o propósito de completeza, apresentamos nesta seção o algoritmo de decodificação, [4], para os empacotamentos esféricos $\mathbb{L}_{N n}$ de $\mathbb{R}^{N n}$ obtidos com a construção multicamada da Seção 2. Este algoritmo pode ser usado para a quantização de vetores e/ou para a decodificação de códigos reticulados, isto é, um subconjunto finito de pontos de um reticulado, para um canal gaussiano. Para maiores detalhes sobre codificações e algoritmos de decodificações de reticulados, [2] ou [3].

Um algoritmo de decodificação por máxima verossimilhança ou simplesmente algoritmo $M L$ para um reticulado $\Gamma$ em $\mathbb{R}^{N}$ é um algoritmo que, dado $\mathrm{x} \in \mathbb{R}^{N}$, encontra um ponto de $\Gamma$ mais próximo de $\mathrm{x}$ do que qualquer outro ponto de $\Gamma$, isto é, dado $\mathrm{x} \in \mathbb{R}^{N}$ existe $\lambda \in \Gamma$ tal que $\mathrm{N}(\mathrm{x}-\lambda) \leq \mathbf{N}\left(\mathbf{x}-\lambda^{\prime}\right)$ para todo $\lambda^{\prime} \in \Gamma, \lambda \neq \lambda^{\prime}$, onde $\mathbf{N}(\mathbf{x})$ denota a norma do vetor $\mathbf{x}$. Assim, uma região de decisão de um algoritmo de decodificação por $\mathrm{ML}$ para $\Gamma$ é essencialmante umn roniãn do Vornoni nara $\Gamma$, calvo amhiguidade envolvida em resolver empate na fronteira. Note que, se $\Phi(\lambda)$ é o ponto mais próximo de $\Gamma$ para $\mathrm{x} \in \mathbb{R}^{N}$, então $\mathrm{r}+\Phi(\lambda-\mathrm{r})$ é o ponto mais próximo de $\mathbf{r}+\Gamma$ para $\mathbf{x} \in \mathbb{R}^{N}$. Portanto, um algoritmo de decodificação por $\mathrm{ML}$ de um reticulado $\Gamma$ pode ser usado como um algoritmo de decodificação por $\mathrm{ML}$ de uma translação de $\Gamma$. Como a medida de distância de um reticulado $\Gamma$ em $\mathbb{R}^{N}$ é invariante por translação temos que o algoritmo por $\mathrm{ML}$ para $\Gamma$ em $\mathbb{R}^{N}$ é invariante por translação.

Um algoritmo de decodificação com distância limitada para um empacotamento esférico $\Gamma$ em $\mathbb{R}^{N}$ é um algoritmo com a seguinte propriedade: dado $\mathrm{x} \in \mathbb{R}^{N}$ se existe $\lambda \in \Gamma$ tal que $\mathbf{N}(x-\lambda)<d_{\min }^{2}(\Gamma) / 4$, então $\mathrm{x}$ será decodificado como $\lambda$. A seguir apresentamos um algoritmo de decodificação com distância limitada ou algoritmo de decodificação por estágio, [4], para os empacotamentos esféricos $\mathbb{L}_{N n}$ em $\mathbb{R}^{N n}$ obtidos através da construção proposta na Seção 2 .

Algoritmo $A^{a}:$ Dado $r \in \mathbb{R}^{n N}$.

Passo 0 - Faça $\mathrm{r}_{0}=\mathrm{r}$ e decodifique $\mathrm{r}_{0}$ no ponto $\left(\widehat{c}_{0} \otimes \mathrm{g}_{0}\right)+$ $\widehat{\lambda}_{1}$ mais próximo do reticulado $\Gamma_{0}=\left(C_{0} \otimes\left[\Lambda_{0} / \Lambda_{1}\right]\right)+$ $\Lambda_{1}^{n}$ em $\mathbb{R}^{n N}$

Passo 1 - Faça $r_{1}=r_{0}-\left(\widehat{c}_{0} \otimes g_{0}\right)$ e decodifique $\mathbb{r}_{1}$ no ponto $\left(\widehat{c}_{1} \otimes g_{1}\right)+\widehat{\lambda}_{2}$ mais próximo do reticulado $\Gamma_{1}=\left(C_{1} \otimes\left[\Lambda_{1} / \Lambda_{2}\right]\right)+\Lambda_{2}^{n}$ em $\mathbb{R}^{n N}$;

Passo $(\mathbf{m}-1)$ - Faça $\mathbf{r}_{m-1}=r_{m-2}-\left(\widehat{c}_{m-2} \otimes g_{m-2}\right)$ e decodifique $\mathbb{r}_{m-1}$ no ponto $\left(\widehat{c}_{m-1} \otimes \mathrm{g}_{m-1}\right)+\widehat{\lambda}_{m}$ mais próximo do reticulado $\Gamma_{m-1}=\left(C_{m-1} \otimes\right.$ $\left.\left[\Lambda_{m-1} / \Lambda_{m}\right]\right)+\Lambda_{m}^{n}$ em $\mathbb{R}^{n N}$.

Portanto, $\mathbf{r}$ será decodificado como $\widehat{l}=\widehat{U} B+\widehat{\lambda}_{m} \in \mathbb{L}_{N n}$, pois $\mathbf{r}_{m-1}=\mathfrak{r}_{0}-\sum_{i=0}^{m-2}\left(\widehat{\mathbf{c}}_{i} \otimes \mathbf{g}_{i}\right)$.

O próximo resultado é uma extensão para o empacotamento esférico $\mathbb{L}_{N n}$ de um resultado semelhante para reticulados em [4], onde omitiremos as demonstrações (veja [13] para maiores detalhes).

Lema 5 Se $q_{i}=q$ e os códigos $C_{i},: 0 \leq i \leq m-1$, são lineares, então o algoritmo $A^{a}$ é invariante por translação.

Teorema 6 Dado $\mathrm{r} \in \mathbb{R}^{N n}$, se $C_{0} \subseteq \cdots \subseteq C_{m-1},: q_{i}=$ $q,: 0 \leq i \leq m-1$, e existe $1 \in \mathrm{L}_{N n}$ tal que $\mathrm{N}(\mathrm{r}-\mathrm{l})<$ $\frac{d_{\min }^{2}\left(\mathbf{L}_{N n}\right)}{4}$, então $\mathbf{r}$ será decodificado corretamente pelo algoritmo $A^{a}$.

O conjunto de pontos $r \in \mathbb{R}^{N n}$ que mapeia para o ponto 0 é chamado a região de decisão, isto é,

$$
R^{a}(\mathbf{0})=\left\{\mathrm{r} \in \mathbb{R}^{N n}: \mathbb{N}(\mathbf{r}) \leq d_{\min }^{2}\left(\mathbb{L}_{N n}\right) / 4\right\}
$$

do algoritmo $A^{a}$. Pelo Lema 5 todas as regiões de decisão $R^{a}(\mathbf{l})$ são congruentes e, $R^{a}(1)=1+R^{a}(0)$ para todo $1 \in \mathbb{L}_{N n}$. Neste caso, $R^{a}(0)$ é uma região fundamental para

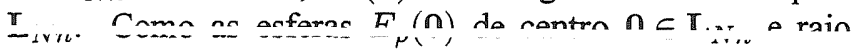
$\rho=d_{\min }\left(\mathbb{L}_{N n}\right) / 2$, devem tocar-se, temos que existem pontos de norma igual a $\rho^{2}$ na fronteira de $R^{a}(\mathbf{0})$. O número de pontos na fronteira de $R^{a}(\mathbf{0})$ com norma igual a $\rho^{2}$ é o coeficiente de erro efetivo de $\mathbb{L}_{N n}$ e, denotaremos por $E_{e}\left(\mathbf{L}_{N n}\right)$. Em geral, $E_{e}\left(\mathbb{L}_{N n}\right) \geq E\left(\mathbb{L}_{N n}\right)$. Assim, $A^{a}$ é um algoritmo subótimo, ou equivalentemente, $A^{a}$ é um algoritmo de decodificação com distância limitada para o empacotamento esférico $\mathbb{L}_{N n}$ em $\mathbb{R}^{N n}$.

O próximo resultado é uma extensão para o reticulado $\mathrm{L}_{N n}$ de um resultado semelhante para reticulados em [4]. Omitiremos a demonstração, porém, a mesma pode ser encontrada em [13].

Teorema $7 \mathrm{Se} C_{0} \subseteq \cdots \subseteq C_{m-1}$ e $q_{i}=q, 0 \leq i \leq m-1$, então o coeficiente de erro efetivo $E_{e}\left(\mathbb{L}_{N n}\right)$ é dado por

$$
E_{e}\left(\mathbb{L}_{N n}\right)=
$$

$$
=\sum_{d_{\min }^{2}\left(\mathbf{L}_{N n}\right)=d_{H}\left(C_{i}\right) d_{\min _{n}}^{2}\left(\Lambda_{i}\right)}\left(E\left(C_{i}\right) E\left(\Lambda_{i+1}\right)\right)^{d_{H}\left(C_{i}\right)}+E_{m}
$$

onde

$$
E_{m}=\left\{\begin{array}{ll}
n E\left(\Lambda_{m}\right), & \text { se }: d_{\min }^{2}\left(\Lambda_{m}\right)=d_{\min }^{2}\left(\mathbb{L}_{N n}\right) \\
0, & \text { se }: d_{\min }^{2}\left(\Lambda_{m}\right)>d_{\min }^{2}\left(\mathbb{L}_{N n}\right)
\end{array} .\right.
$$




\section{Antonio de Andrade e Silva e Reginaldo Palazzo Jr. Construção de Empacotamentos Esfiéricos Via Códigos de Classes Laterais Generalizados}

A seguir, iremos apresentar o cálculo aproximado da complexidade de decodificação do algoritmo $A^{a}$ para o empacotamento esférico $\mathbb{L}_{N n}$. Para maiores detalhes e exemplos, ver [4].

Primeiro. Dado $\mathbf{r}_{i}=\left(\mathbf{r}_{i 1}, \ldots, \mathbf{r}_{i n}\right), \mathbf{r}_{i j} \in \mathbb{R}^{N}, 0 \leq i \leq$ $m-1,1 \leq j \leq n$, então para cada $i=0, \ldots, m-1$, o algoritmo escolhe um $c_{i j} \in \mathbb{N}_{q_{i}}$ que esteja mais próximo de cada uma das componentes $\mathbf{r}_{i j}$ de $\mathbf{r}_{i}, 1 \leq j \leq n$, isto é,

$$
d_{i j} \triangleq \min \left\{\mathrm{N}\left(\mathrm{r}_{i j}-\left(\left(c_{i j} \mathbf{g}_{i}\right)+\lambda_{(i+1) j}\right)\right)\right\} \text {. }
$$

Note que $d_{i j}$ é a mínima distância euclidiana quadrática de cada classe lateral à esquerda de $\Lambda_{i+1}$ em $\Lambda_{i}$ da componente recebida $\mathbf{r}_{i j}, 1 \leq j \leq n$.

Segundo. Para cada $i=0, \ldots, m-1$, seja $\tilde{\mathbf{c}}_{i}=$ $\left(\tilde{c}_{i 1}, \ldots, \tilde{c}_{i n}\right)$ uma aproximação para a palavra-código $\mathbf{c}_{i}=$ $\left(c_{i 1}, \ldots, c_{i n}\right) \in C_{i}$. Então um algoritmo de decodificação suave para decodificar $C_{i}$, (Apêndice de [7] ou [15]), é usado para corrigir erros em $\tilde{\mathbf{c}}_{i}$, e a palavra resultante é uma palavracódigo $\hat{\mathbf{c}}_{i} \in C_{i}$. isto é,

$$
d_{i} \triangleq \min \left\{\mathbf{N}\left(\mathbf{r}_{i}-\left(\left(\mathbf{c}_{i} \otimes \mathbf{g}_{i}\right)+\lambda_{i+1}\right)\right)\right\} .
$$

Como a norma é uma medida de distância aditiva e

$$
\min \left(\sum_{i=0}^{m-1} X_{i}\right)=\sum_{i=0}^{m-1}\left(\min X_{i}\right)
$$

temos que

$$
d_{i}=\sum_{j=1}^{n} d_{i j}, 0 \leq i \leq m-1 .
$$

Finalmente, como o algoritmo $A^{a}$ para $\mathbb{L}_{N n}$ usa apenas a palavra código $\mathrm{c}_{i} \in C_{i}$ para minimizar $d_{i} \mathrm{e}$

$$
\begin{aligned}
\Gamma_{i} & =\left\{\mathbf{c}_{i} \otimes \mathbf{g}_{i}: \mathbf{c}_{i} \in C_{i}\right\}+\Lambda_{i+1}^{n}=\bigcup_{\mathbf{c}_{i} \in C_{i}}\left(\mathbf{c}_{i} \otimes \mathrm{g}_{i}+\Lambda_{i+1}^{n}\right) \\
& =\bigcup_{\mathbf{c}_{i} \in C_{i}}\left\{\left(c_{i 1} \mathbf{g}_{i}+\lambda_{i 1}, \ldots, c_{i n} \mathbf{g}_{i}+\lambda_{i n}\right): \lambda_{i j} \in \Lambda_{i+1}\right\},
\end{aligned}
$$

e $\Lambda_{i}=T_{2}^{i}\left(\Lambda_{0}\right), i=1,2$, os subreticulados de $\Lambda_{0}$ induzidos pelo endomorfismo $T_{2}$ de $\Lambda_{0}$ definido pela matriz

$0 \leq i \leq m-1$, a complexidade para decodificar $\Gamma_{i}$ é dada aproximadamente por

$$
\mathbb{N}_{D}\left(\Gamma_{i}\right)=n\left(\mathbb{N}_{D}\left(\Lambda_{i} / \Lambda_{i+1}\right)+1\right)+\mathbb{N}_{D}\left(C_{i}\right),
$$

onde $\mathrm{N}_{D}\left(\Lambda_{i} / \Lambda_{i+1}\right)$ é a complexidade do algoritmo $\mathrm{ML}$ usado para decodificar a partição $\Lambda_{i} / \Lambda_{i+1}$, (Apêndice [7]), e $\mathrm{N}_{D}\left(C_{i}\right)$ é a complexidade do algoritmo com decisão suave por ML usado para decodificar $C_{i}$. Portanto, a complexidade para o algoritmo $A^{a}$ para $\mathbb{L}_{N n}$ é dada por

$$
\mathbb{N}_{D}^{a}\left(\mathbb{L}_{N n}\right) \triangleq \sum_{i=0}^{m-1} \mathbb{N}_{D}\left(\Gamma_{i}\right)+m n N
$$

onde $m n N$ refere-se aos passos do algoritmo $A^{a}$ que calcula cada $\mathbb{I}_{i}$ a ser decodificado em relação a $\Gamma_{i}$, no passo subsequente.

A complexidade normalizada é dada por

$$
\tilde{\mathbb{N}}_{D}^{a}\left(\mathbb{L}_{N n}\right)=\frac{2}{n N} \mathbb{N}_{D}^{a}\left(\mathbb{L}_{N n}\right)
$$

$$
\left[T_{2}\right]=\left[\begin{array}{rr}
1 & 1 \\
1 & -1
\end{array}\right]
$$

A relação de degradação de desempenho em $[d B]$ por redução de complexidade em [oitavas], [4], do reticulado $\mathbb{L}_{N n}$ é dada por

$$
\beta\left(\mathbb{L}_{N n}\right) \triangleq-\frac{\Delta\left(\mathbb{L}_{N n}\right)}{\log _{2}\left(\frac{\mathbf{N}_{D}^{a}\left(\mathbb{L}_{N n}\right)}{\mathbf{N}_{D}\left(\mathbb{L}_{N n}\right)}\right)}
$$

$$
\text { e } \Delta\left(\mathbb{L}_{N n}\right) \approx 0.22 \log _{2}\left(\frac{E_{e}\left(\mathbf{L}_{N n}\right)}{E\left(\mathbb{L}_{N n}\right)}\right)
$$

Note que um algoritmo de decodificação misto pode alcançar o melhor compromisso entre desempenho e complexidade, isto é, no primeiro passo do algoritmo $A^{a}$ usamos um decodificador com decisão suave por $\mathrm{ML}$ e um decodificador com decisão abrupta subótimo nos passos subsequentes. Para comparações do algoritmo $A^{a}$ com outros algoritmos, ver [3].

\section{EXEMPLOS}

Nesta seção apresentaremos alguns exemplos de empacotamentos esféricos como ilustrações da construção multicamada da Seção 2, bem como a obtenção de seus principais parâmetros. Para mais exemplos e comparações, ver [4].

Exemplo 8 Seja $\Lambda_{0} / \Lambda_{1} / \Lambda_{2}$ uma cadeia de partições, com 2-níveis e 2-maneiras, onde $\Lambda_{0}=\mathbb{Z}^{2}$ é o reticulado cúbico em $\mathbb{R}^{2}$ com uma matriz geradora

$$
M_{0}=\left[\begin{array}{ll}
1 & 0 \\
0 & 1
\end{array}\right]
$$

que escala $\Lambda_{0}$ por $\sqrt{2}$ e rotaciona por $45^{\circ}$. Neste caso,

$\mathrm{g}_{0}=(1,0), \quad \mathrm{g}_{1}=(1,1), \quad d_{\min }^{2}\left(\mathbb{L}_{2 n}\right)=$ $\min _{0 \leq i \leq 1}\left\{2^{i} d_{H}\left(C_{i}\right), 4\right\}, V\left(\mathbb{L}_{2 n}\right)=2^{2 n-k} e$ $\gamma\left(\mathbf{L}_{2 n}\right)=2^{\frac{k-2 n}{n}} d_{\min }^{2}\left(\mathbf{L}_{2 n}\right)$, onde $k=k_{0}+k_{1}$.

Usaremos a notação $[n, k, d]_{q}$ para um código sobre $G F(q)$, com comprimento $n$, dimensão $k$ e distância de Hamming $d$.

(1) Para os códigos $C_{0}=[2,1,2]_{2}$ e $C_{1}=[2,2,1]_{2}$, Tabela-I de [14], temos

$$
d_{\min }^{2}\left(\mathbb{L}_{4}\right)=2, V\left(\mathbb{L}_{4}\right)=2: e: \gamma\left(\mathbb{L}_{4}\right)=1.51[d B],
$$

$$
\begin{aligned}
& \mathbb{E}\left(\mathbf{L}_{4}\right)=\sum_{w_{H}\left(\mathfrak{c}_{0}\right)=d_{H}\left(C_{0}\right)} M^{(0)}\left(\mathfrak{c}_{0}\right)+ \\
& +\sum_{w_{H}\left(\mathfrak{c}_{1}\right)=d_{H}\left(C_{1}\right)} M^{(1)}\left(\mathfrak{c}_{1}\right) \\
& =M^{(0)}\left(\mathbb{c}_{0}\right)+2 M^{(1)}\left(\mathfrak{c}_{1}\right) .
\end{aligned}
$$


Para $\mathbf{c}_{0}=(11)$, obtemos

$$
\begin{aligned}
& M^{(0)}\left(\mathbf{c}_{0}\right)= \\
& =\sum_{\substack{\mathbf{c}_{1} \in C_{1} \\
w_{H}\left(\mathbf{c}_{1} * \mathbf{c}_{0}\right)=w_{H}\left(\mathbf{c}_{1}\right)}}^{2} \prod_{k=1}^{2}\left[E^{(0)}\left(c_{o k} \mathrm{~g}_{0}+c_{1 k} \mathrm{~g}_{1}+\Lambda_{2}\right)\right] \\
& =E^{(0)}\left(\mathrm{g}_{0}+\Lambda_{2}\right)^{2}+2 E^{(0)}\left(\mathrm{g}_{0}+\Lambda_{2}\right) \times \\
& E^{(0)}\left(\mathrm{g}_{0}+\mathrm{g}_{1}+\Lambda_{2}\right)+E^{(0)}\left(\mathrm{g}_{0}+\mathrm{g}_{1}+\Lambda_{2}\right)^{2} \\
& =2^{2}+2.2 .2+2^{2}=16 .
\end{aligned}
$$

$e$

$$
M^{(1)}\left(\mathbf{c}_{1}\right)=E^{(1)}\left(\Lambda_{2}\right)^{d_{H}\left(C_{1}\right)}=4^{1}=4
$$

Logo,

$$
\begin{aligned}
& E\left(\mathbf{L}_{4}\right)=24:: e:: \widetilde{E}\left(\mathbb{L}_{4}\right)=12 . \\
& E_{e}\left(\mathbf{L}_{4}\right)=E^{(0)}\left(C_{0}\right) E^{(0)}\left(\Lambda_{1}\right)^{d_{H}\left(C_{0}\right)}+ \\
& E^{(1)}\left(C_{1}\right) E^{(1)}\left(\Lambda_{2}\right)^{d_{H}\left(C_{1}\right)} \\
& =4^{2}+2.4^{1}=24:: e:: \widetilde{E}_{e}\left(\mathbb{L}_{4}\right)=12 .
\end{aligned}
$$

Finalmente,

$$
\begin{aligned}
& \mathbf{N}_{D}^{a}\left(\mathbf{L}_{4}\right)=2\left(\mathbf{N}_{D}\left(\Lambda_{0} / \Lambda_{1}\right)+1\right)+\mathbf{N}_{D}\left(C_{0}\right)+ \\
& +2\left(\mathbf{N}_{D}\left(\Lambda_{1} / \Lambda_{2}\right)+1\right)+\mathbf{N}_{D}\left(C_{1}\right)+8 \\
& =6+4+6+2+8=26
\end{aligned}
$$

Note que $\mathrm{L}_{4}$ é similar ao reticulado do tabuleiro de xadrez $\mathrm{D}_{4}$ em $\mathbb{R}^{4},[2]$.

(2) Para os códigos $C_{0}=[4,1,4]_{2}$ e $C_{1}=[4,3,2]_{2}$, Tabela-I de [14], obtemos

$$
\begin{gathered}
d_{\min }^{2}\left(\mathbf{L}_{8}\right)=4,: V\left(\mathbb{L}_{8}\right)=2^{4} \\
\gamma\left(\mathbb{L}_{8}\right)=3,01[d B],: \widetilde{E}\left(\mathbb{L}_{8}\right)=60,: \widetilde{E}_{e}\left(\mathbf{L}_{8}\right)=92
\end{gathered}
$$

$$
e:: \mathbb{N}_{D}^{a}\left(\mathbb{L}_{8}\right)=52 \text {. }
$$

Como $\mathbf{N}_{D}\left(\mathbf{L}_{8}\right)=72$, [2], temos que a relação de degradação de desempenho em $[d B]$ por redução de complexidade em [oitavas] do reticulado $\mathbb{L}_{8}$ é dada por

$$
\beta\left(\mathbf{L}_{8}\right)=0.29 \text {. }
$$

Note que $\mathbf{L}_{8}$ é similar ao reticulado de Gosset $\mathbb{E}_{8}$ em $\mathbb{R}^{8},[2]$.

Exemplo 9 Seja $\Lambda_{0} / \Lambda_{1} \cdots / \Lambda_{m}$ uma cadeia de partições, com m-niveis e 3- elou 4-maneiras, onde $\Lambda_{0}=\mathrm{A}_{2}$ é o reticulado hexagonal em $\mathbb{R}^{2}$ com uma matriz geradora

$$
M_{0}=\left[\begin{array}{cc}
1 & -\frac{1}{2} \\
0 & \frac{\sqrt{3}}{2}
\end{array}\right]
$$

e $\Lambda_{i}=T_{3}^{i}\left(T_{4}^{j}\left(\Lambda_{0}\right)\right), i, j=0,1, \ldots, m$, os subreticulados de $\Lambda_{0}$ induzidos pelos endomorfismos $T_{3}$ e $T_{4}$ de $\Lambda_{0}$ definidos pelas matrizes, [2],

$$
\left[T_{3}\right]=\left[\begin{array}{rr}
\frac{3}{2} & \frac{\sqrt{3}}{2} \\
-\frac{\sqrt{3}}{2} & \frac{3}{2}
\end{array}\right]::: e:::\left[T_{4}\right]=\left[\begin{array}{ll}
2 & 0 \\
0 & 2
\end{array}\right] .
$$

Usando as cadeias de partições do reticulado hexagonal $A_{2}$, reproduzimos todos os empacotamentos esféricos da Tabela-VIII de [9]. Como um exemplo, seja $\Lambda_{0} / \Lambda_{1} / \Lambda_{2}$ uma cadeia de partições, com 2-níveis, onde $\Lambda_{0}=\mathrm{A}_{2}, \Lambda_{1}=$ $T_{4}\left(\Lambda_{0}\right)$ e $\Lambda_{2}=T_{3}\left(\Lambda_{1}\right)$. Então para os códigos $C_{0}=$ $[18,9,8]_{4}, C_{0}^{\prime}=[30,15,12]_{4}$, Tabela-VI de $[9]$, e $C_{1}=$ $[18,17,2]_{3}, C_{1}^{\prime}=[30,26,3]_{3}$, Tabela-V de [9], obtemos os empacotamentos esféricos $\mathbb{L}_{36}$ e $\mathbb{L}_{60}$, que são os mais densos em suas dimensões.

Seja $\Lambda_{0} / \Lambda_{1} \cdots / \Lambda_{m}$ uma cadeia de partições, com $m$ níveis e 4-maneiras, onde $\Lambda_{0}=D_{4}$ é o reticulado do tabuleiro de xadrez em $\mathbb{R}^{4}$ com uma matriz geradora

$$
M_{0}=\left[\begin{array}{llll}
2 & 1 & 1 & 1 \\
0 & 1 & 0 & 0 \\
0 & 0 & 1 & 0 \\
0 & 0 & 0 & 1
\end{array}\right]
$$

e $\Lambda_{i}=T_{4}^{i}\left(\Lambda_{0}\right), i=0,1, \ldots, m$, os subreticulados de $\Lambda_{0}$ induzidos pelo endomorfismo $T_{4}$ de $\Lambda_{0}$ definido pela matriz

$$
\left[T_{4}\right]=\left[\begin{array}{rrrr}
1 & 1 & 0 & 0 \\
1 & -1 & 0 & 0 \\
0 & 0 & 1 & 1 \\
0 & 0 & 1 & -1
\end{array}\right]
$$

Usando os melhores códigos quaternários conhecidos, Tabela-VI de [9], e a cadeia de partições do reticulado $\mathbf{D}_{4}$, obtemos uma variedade de empacotamentos esféricos mostrados na Tabela 1 , onde os códigos $C_{i}=\left[n, k_{i}, d_{i}\right]_{4}$ nas quatro colunas da tabela são provenientes da Tabela-VI de [9]. Na quinta coluna, está o ganho de codificação fundamental $\gamma\left(\mathbf{L}_{N n}\right)$, em $[d B]$, dos empacotamentos propostos e na sexta coluna $\gamma c s$ e $\gamma k p$ representam o ganho de codificação fundamental, em $[d B]$, dos melhores empacotamentos da Tabela 3.1 de [2] e da Tabela-VIII de [9], respectivamente. Finalmente, na sétima coluna temos a dimensão dos empacotamentos $\mathbb{L}_{N n}$.

Nas dimensões 68 e 72, os empacotamentos esféricos obtidos (Tabela 1) são mais densos do que os correspondentes da Tabela VIII de [9]. As densidades de centros destes empacotamentos esféricos são $\delta\left(\mathbb{L}_{68}\right) \approx 2^{24.95}$ e $\delta\left(\mathbb{L}_{72}\right) \approx 2^{30.73}$, respectivamente. Os empacotamentos esféricos obtidos com esta construção dependem muito dos códigos e dos endomorfismos a serem utilizados, uma vez que a utilização de códigos com distâncias de Hamming maiores para os primeiros níveis da partição implica em melhores empacotamento dos subcódigos para um endomorfismo apropriado. Para maiores detalhes veja [13]. 


\begin{tabular}{|llll|c|l|l|}
\hline $\mathbf{C}_{0}$ & $\mathbf{C}_{1}$ & $\mathbf{C}_{2}$ & $\mathbf{C}_{3}$ & $\gamma\left(\overline{\mathbf{L}}_{N n}\right)[d B]$ & $\gamma[d B]$ & $N n$ \\
\hline$[2,1,2]$ & {$[2,2,1]$} & {$[2,2,1]$} & {$[2,2,1]$} & 3.01 & $3.01 \mathrm{cs}$ & $\mathbf{L}_{8}$ \\
\hline$[3,2,2]$ & {$[3,3,1]$} & {$[3,3,1]$} & {$[3,3,1]$} & 3.51 & $3.63 \mathrm{cs}$ & $\mathbf{L}_{12}$ \\
\hline$[4,1,4]$ & {$[4,3,2]$} & {$[4,4,1]$} & {$[4,4,1]$} & 4.52 & $4.52 \mathrm{cs}$ & $\mathbf{L}_{16}$ \\
\hline$[5,2,4]$ & {$[5,4,2]$} & {$[5,5,1]$} & {$[5,5,1]$} & 5.12 & $5.12 \mathrm{cs}$ & $\mathbf{L}_{20}$ \\
\hline$[6,3,4]$ & {$[6,5,2]$} & {$[6,6,1]$} & {$[6,6,1]$} & 5.52 & $6.02 \mathrm{cs}$ & $\mathbf{L}_{24}$ \\
\hline$[7,3,4]$ & {$[7,6,2]$} & {$[7,7,1]$} & {$[7,7,1]$} & 5.38 & $5.81 \mathrm{cs}$ & $\mathbf{L}_{28}$ \\
\hline$[8,1,8]$ & {$[8,4,4]$} & {$[8,7,2]$} & {$[8,8,1]$} & 6.02 & $6.28 \mathrm{cs}$ & $\mathbf{L}_{32}$ \\
\hline$[9,1,9]$ & {$[9,5,4]$} & {$[9,8,2]$} & {$[9,9,1]$} & 6.19 & $6.38 \mathrm{kp}$ & $\mathbf{L}_{36}$ \\
\hline$[10,2,8]$ & {$[10,6,4]$} & {$[10,9,2]$} & {$[10,10,1]$} & 6.62 & $6.62 \mathrm{cs}$ & $\mathbf{L}_{40}$ \\
\hline$[11,2,8]$ & {$[11,7,4]$} & {$[11,10,2]$} & {$[11,11,1]$} & 6.71 & $6.98 \mathrm{cs}$ & $\mathbf{L}_{44}$ \\
\hline$[12,3,8]$ & {$[12,8,4]$} & {$[12,11,2]$} & {$[12,12,1]$} & 7.02 & $7.78 \mathrm{cs}$ & $\mathbf{L}_{48}$ \\
\hline$[13,4,8]$ & {$[13,9,4]$} & {$[13,12,2]$} & {$[13,13,1]$} & 7.30 & $7.30 \mathrm{kp}$ & $\mathbf{L}_{52}$ \\
\hline$[14,5,8]$ & {$[14,10,4]$} & {$[14,13,2]$} & {$[14,14,1]$} & 7.53 & $7.52 \mathrm{kp}$ & $\mathbf{L}_{56}$ \\
\hline$[15,6,8]$ & {$[15,11,4]$} & {$[15,14,2]$} & {$[15,15,1]$} & 7.73 & $7.77 \mathrm{kp}$ & $\mathbf{L}_{60}$ \\
\hline$[16,1,16]$ & {$[16,7,8]$} & {$[16,12,4]$} & {$[16,15,2]$} & 8.10 & $8.10 \mathrm{cs}$ & $\mathbf{L}_{64}$ \\
\hline$[17,1,17]$ & {$[17,8,8]$} & {$[17,13,4]$} & {$[17,16,2]$} & 8.23 & $8.01 \mathrm{kp}$ & $\mathbf{L}_{68}$ \\
\hline$[18,1,18]$ & {$[18,9,8]$} & {$[18,13,4]$} & {$[18,17,2]$} & 8.59 & $8.16 \mathrm{kp}$ & $\mathbf{L}_{72}$ \\
\hline$[19,9,8]$ & {$[19,14,4]$} & {$[19,18,2]$} & {$[19,19,1]$} & 8.16 & $8.24 \mathrm{kp}$ & $\mathbf{L}_{76}$ \\
\hline$[20,2,16]$ & {$[20,10,8]$} & {$[20,15,4]$} & {$[20,19,2]$} & 8.43 & $8.73 \mathrm{cs}$ & $\mathbf{L}_{80}$ \\
\hline$[21,3,16]$ & {$[21,10,8]$} & {$[21,16,4]$} & {$[21,20,2]$} & 8.53 & $8.54 \mathrm{kp}$ & $\mathbf{L}_{84}$ \\
\hline$[22,3,16]$ & {$[22,11,8]$} & {$[20,17,4]$} & {$[22,21,2]$} & 8.62 & $8.58 \mathrm{kp}$ & $\mathbf{L}_{88}$ \\
\hline$[23,3,16]$ & {$[23,12,8]$} & {$[23,18,4]$} & {$[23,22,2]$} & 8.70 & $8.72 \mathrm{kp}$ & $\mathbf{L}_{92}$ \\
\hline$[24,4,16]$ & {$[24,13,8]$} & {$[24,19,4]$} & {$[24,23,2]$} & 8.91 & $9.29 \mathrm{cs}$ & $\mathbf{L}_{96}$ \\
\hline$[25,5,16]$ & {$[25,14,8]$} & {$[25,20,4]$} & {$[25,24,2]$} & 9.09 & $9.07 \mathrm{kp}$ & $\mathbf{L}_{100}$ \\
\hline
\end{tabular}

Tabela 1. Empacotamentos esféricos obtidos de partições do reticulado $\mathbb{D}_{4}$.

\section{CONCLUSÕES}

Neste trabalho foi proposta uma construção multinível para a obtenção de empacotmentos esféricos ou reticulados com expressões explícitas para os seus principais parâmetros. Os empacotamentos esféricos obtidos com esta construção dependem dos códigos e dos endomorfismos a serem utilizados, uma vez que a utilização de códigos com distâncias de Hamming maiores para os primeiros níveis da partição implica em melhores empacotamento dos subcódigos para um endomorfismo apropriado. Nas dimensões 68 e 72, um novo recorde de densidades foi alcançado.

\section{REFERÊNCIAS}

[1] A.R. Calderbank and N.J.A. Sloane, "New trellis codes based on lattices and cosets," IEEE Trans. Inform. Theory, vol. 33, pp. 177-195, 1987.

[2] J.H. Conway and N.J.A. Sloane, Sphere Packing, Lattices and Groups, New York, Springer-Verlag, 1988.

[3] M.A.O. Costa e Silva, Reticulados e suas Partições Aplicados a Codificação sobre Canais AWGN Limitados em Faixa, Tese de Doutorado, FEE-UNICAMP, 1991.

[4] M.A.O. Costa e Silva e R. Palazzo, Jr., "A boundeddistance decoding algorithm for lattices based on generalized code formula," IEEE Trans. Inform. Theory, vol. 40, pp. 2075-2082, 1994.
[5] D.S. Dummit and R.M. Foote, Abstract Algebra, New

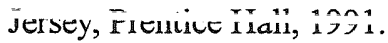

[6] G.D. Forney, Jr., "Coset codes I: Introduction and geometrical classification," IEEE Trans. Inform. Theory, vol. 34, pp. 1123-1151, 1988.

[7] G.D. Forney, Jr., "Coset codes II: Binary lattices and related codes," IEEE Trans. Inform. Theory, vol. 34, pp. 1152-1187, 1988.

[8] G.D. Forney, Jr., "A bounded-distance decoding algorithm for the Leech lattices, with generalizations," IEEE Trans. Inform. Theory, vol. 35, pp. 906-909, 1989.

[9] F.R.Kschischang and S. Pasupathy, "Some ternary and quaternary codes and associated sphere packings," IEEE Trans. Inform. Theory, vol. 38, pp. 227-246, 1992.

[10] J. Leech and N.J.A. Sloane, "Sphere packings and error correcting codes," Canad. J. Math., vol. 23, pp. 718745, 1971.

[11] F.J. MacWilliams and N.J.A. Sloane, The Theory of Error-Correcting Codes. New York: North-Holland, 1977.

[12] A.A. e Silva e R. Palazzo, Jr., "Construção de reticulados via fórmula de códigos p-ários generalizado," XIII Simpósio Brasileiro de Telecomunicações, Águas de Lindóia, São Paulo, 1995.

[13] A.A. e Silva, Uma Contribuição à Classe dos Códigos Geometricamente Uniformes, Tese de Doutorado, FEEC-UNICAMP, 1996. 
[14] T. Verhoeff, "An updated table of minimum-distance bounds for binary codes, IEEE Trans. Inform. Theory, vol. 33, pp. 665-680, 1987.
[15] J.K. Wolf, "Efficient maximum likelihood decoding of linear block codes using trellis," IEEE Trans. Inform. Theory, vol. 24, pp. 76-80, 1978. 